\title{
Brief
}

\section{RING plus STRING: Papillary muscle repositioning as an adjunctive repair technique for ischemic mitral regurgitation}

\author{
Frank Langer, MD, and Hans-Joachim Schäfers, MD, Homburg, Germany
}

Supplemental material is available online.
From the Department of Thoracic and Cardiovascular Surgery, University Hospital Homburg, Homburg, Germany.

Received for publication March 4, 2006; accepted for publication April 24, 2006.

Address for reprints: Hans-Joachim Schäfers, MD, Department of Thoracic and Cardiovascular Surgery, University Hospital Homburg, 66421 Homburg, Germany (E-mail: chhjsc@uniklinik-saarland.de).

J Thorac Cardiovasc Surg 2007;133:247-9

$0022-5223 / \$ 32.00$

Copyright @ 2007 by The American Association for Thoracic Surgery

doi:10.1016/j.jtcvs.2006.04.059
I schemic mitral regurgitation (IMR), a frequent complication of myocardial infarction associated with poor prognosis, is increasingly considered to be a ventricular disease rather than a valvular disease. Although few groups report encouraging results after undersized ring annuloplasty (RING), residual/recurrent mitral regurgitation is seen in up to $30 \%$ of patients in most other centers. ${ }^{1}$

Assessment of the mechanism of IMR by means of transesophageal echocardiography (TEE) exhibits restrictive leaflet motion (Carpentier type IIIb) with severe leaflet tethering and displacement of the coaptation point toward the ventricle-geometric distortions that are commonly described as "tenting" of the mitral valve. The height of this tent (ie, the end-systolic distance between the annular plane and the coaptation point) has been used by Calafiore and colleagues ${ }^{2}$ as the key parameter to decide between repair or replacement. If this distance exceeded $10 \mathrm{~mm}$, patients were considered poor repair candidates, and valve replacement was performed.

Several adjunctive repair techniques have been proposed, but none of these techniques has received widespread clinical acceptance. Recently, Kron and associates ${ }^{3}$ proposed relocation of the displaced posterior papillary muscle (PPM) with a transventricular suture anchoring the PPM toward the mitral annulus as an adjunct to RING. Based on this report and the mechanistic insight reported by Tibayan and coworkers ${ }^{4}$ that the distance between the midseptal fibrous annulus and the PPM plays a key role in the pathogenesis of IMR, the direction of internal repositioning of the PPM was refined in a sheep model of acute IMR. ${ }^{5}$ Building on these data, we report a new clinical repair approach (Figure 1) that allows successful repositioning of the PPM under TEE guidance in the beating heart.

\section{Technique}

In patients with IMR and tenting height exceeding $10 \mathrm{~mm}$ (Figure 2, $A$; Figure E1), we added our new adjunctive repair technique to standard mitral valve repair and coronary artery bypass grafting (approved by a local ethics committee and informed consent obtained). After completion of undersized RING, a horizontal aortotomy is performed. Retraction of the right coronary cusp with a retractor allows excellent exposure of the anterior mitral leaflet and both papillary muscles (Figure 1, A). A Teflon-pledgeted 4-0 polytetrafluoroethylene suture (STRING) is passed through the head of the PPM and then passed from the left ventricular cavity through the midseptal fibrous annulus (saddle horn) to be exteriorized through the aortic wall underneath the commissure between the noncoronary and left coronary aortic cusps (Figures 1, $A, B$ ). During termination of CPB, the STRING suture is tied under TEE guidance in the loaded beating heart (Figures E2, E3; mitral regurgitation data are shown in Table E1: tenting height, $15 \pm 3 \mathrm{~mm}$ preoperatively and $6 \pm 2 \mathrm{~mm}$ postoperatively; distance from PPM tip to fibrosa: $44 \pm 4 \mathrm{~mm}$ preoperatively and $36 \pm 2 \mathrm{~mm}$ postoperatively).

This new approach (RING plus STRING) has been used in 12 patients (64 \pm 9 years of age; ejection fraction, $29 \% \pm 6 \%$ ). Surgical intervention included an undersized RING (partial flexible ring implants; average size, $28 \mathrm{~mm}$ ) combined with coronary artery bypass grafting (average, $2.6 \pm 1.2$ grafts). Within short-term follow-up (mean, 12 months; range, 4-16 months), valve function has remained stable, with mitral regurgitation $<$ class II in all patients. 

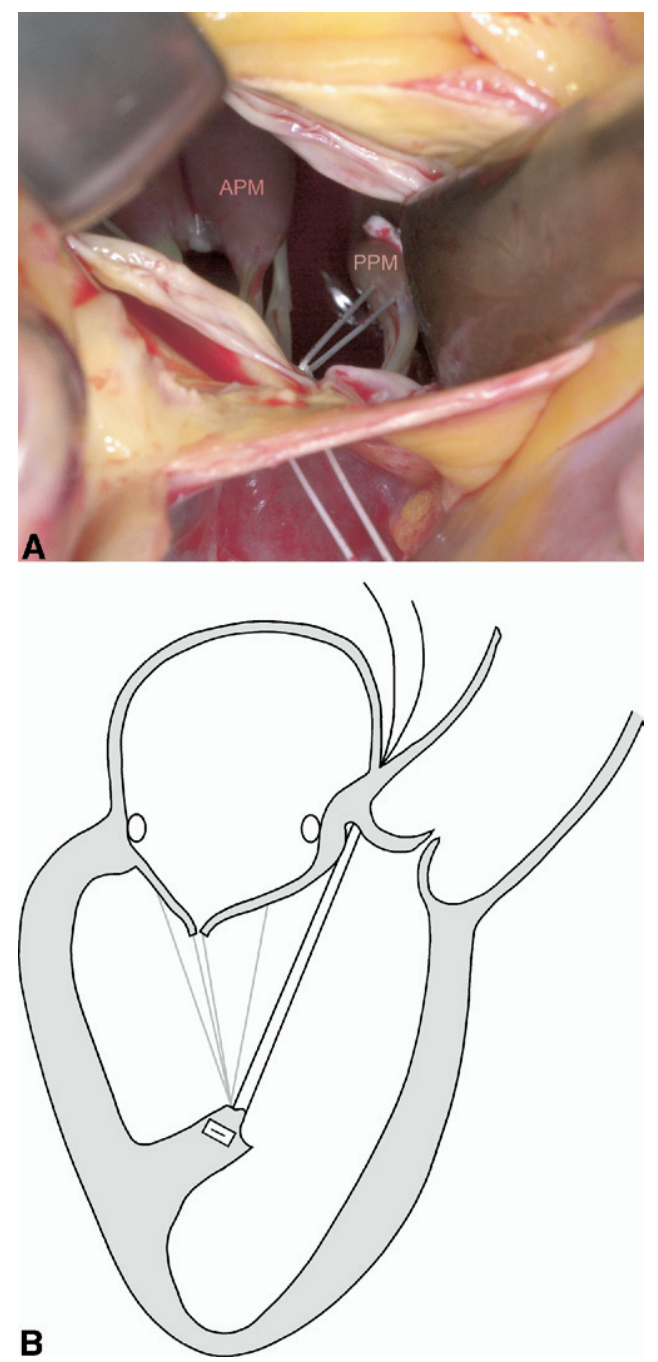

Figure 1. A, Intraoperative photograph of the transventricular suture technique (STRING). A horizontal aortotomy allows excellent exposure of the subvalvular mitral apparatus. A Teflon-pledgeted 4-0 polytetrafluroethylene suture is anchored in the head of the posterior papillary muscle (PPM). The suture is then passed through the fibrosa (midseptal annular saddle horn) under direct vision and exteriorized through the aortic wall underneath the commissure between the noncoronary and left coronary aortic cusps. APM, Anterior papillary muscle. B, Schematic representation of the transventricular suture technique (STRING). The suture is tied under echocardiographic guidance in the loaded beating heart to reposition the displaced posterior papillary muscle toward the fibrosa.

\section{Discussion}

Tenting has been identified as a predictor of severity of IMR and is determined by the distance between the PPM and the fibrosa. Relocation of the displaced PPM toward the fibrosa might thus aid in elimination of residual mitral regurgitation after RING but might also prevent recurrent mitral regurgitation in the deleterious continued remodeling process, which leaves the left ventricle as a
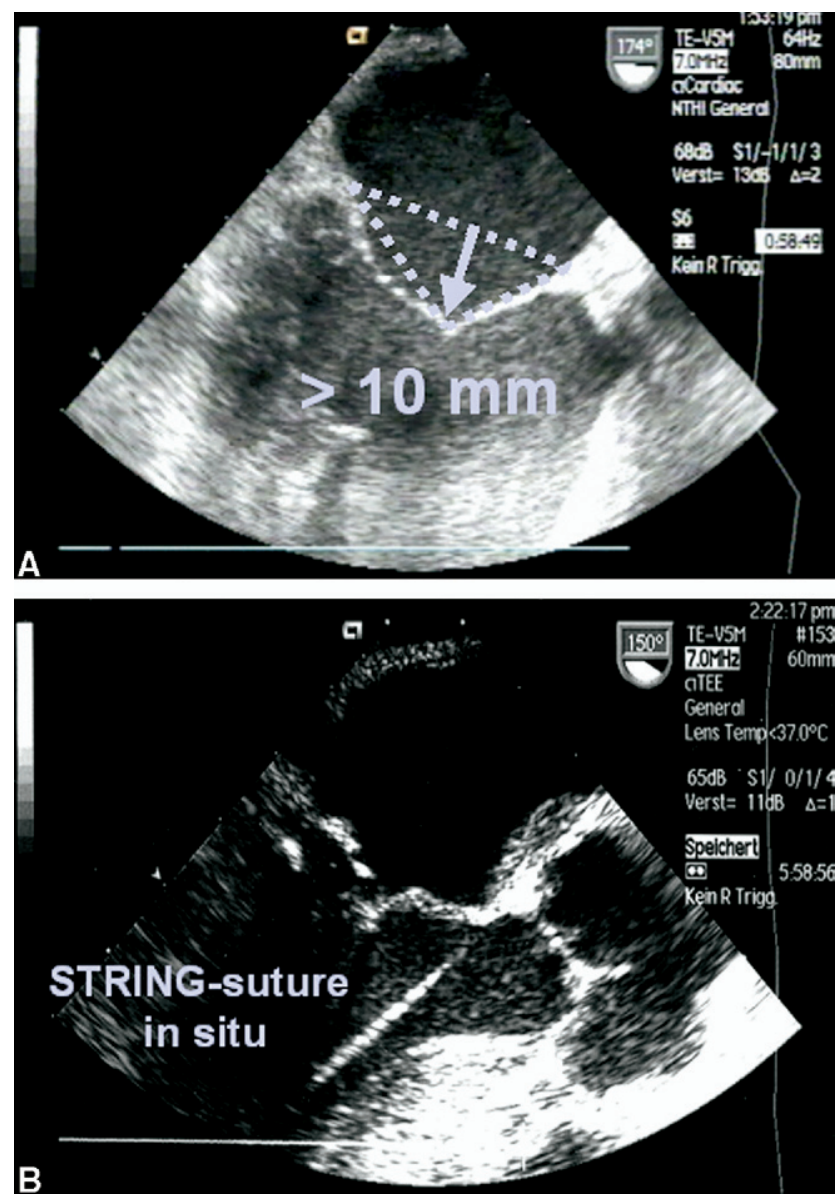

Figure 2. A, Assessment of tenting height (ie, end-systolic orthogonal distance between the annular plane and the coaptation point of both mitral leaflets) by means of intraoperative transesophageal echocardiography. B, Intraoperative transesophageal echocardiography after repair and termination of cardiopulmonary bypass with a transventricular polytetrafluoroethylene suture (STRING) in situ, resulting in normalized morphology of the mitral valve.

moving target. The transventricular STRING suture, which is equivalent to a third-order chord, might function as a seatbelt for the left ventricle, limiting deleterious remodeling.

Anchoring the suture in the fibrosa from the atriotomy approach implies the risk of aortic regurgitation if an aortic cusp is inadvertently involved. A horizontal aortotomy facilitates exposure of the subvalvular apparatus and also allows anchoring of the suture in the midseptal annulus under direct vision. The final tension on the STRING suture is determined by tying under TEE guidance in the loaded beating heart.

Which patients will exhibit residual/recurrent mitral regurgitation after RING? Calafiore and colleagues ${ }^{2}$ distinguished between good and poor repair candidates based on tenting height. If tenting height exceeded $10 \mathrm{~mm}$, valve replacement was performed. We have adopted this arbitrary cutoff point; however, we used this distance to distinguish between RING and RING plus STRING. 
Based on short-term results, we can only speculate on the longevity of this new repair approach. However, placement of artificial primary chordae with polytetrafluoroethylene sutures has been associated with encouraging results in our experience. The excursion of the anterior mitral leaflet has not been limited by the STRING suture in the previous experimental setting ${ }^{5}$ or in our clinical observations. Long-term follow-up is needed to prove the clinical role of this new repair option for patients with IMR.

\section{References}

1. McGee EC, Gillinov AM, Blackstone EH, Rajeswaran J, Cohen G, Najam F, et al. Recurrent mitral regurgitation after annuloplasty for functional ischemic mitral regurgitation. $J$ Thorac Cardiovasc Surg. 2004;128:916-24.

2. Calafiore AM, Di Mauro M, Gallina S, Di Giammarco G, Iaco AL, Teodori G, et al. Mitral valve surgery for chronic ischemic mitral regurgitation. Ann Thorac Surg. 2004;77:1989-97.

3. Kron IL, Green GR, Cope JT. Surgical relocation of the posterior papillary muscle in chronic ischemic mitral regurgitation. Ann Thorac Surg. 2002;74:600-1.

4. Tibayan FA, Rodriguez F, Zasio MK, Bailey L, Liang D, Daughters GT, et al . Geometric distortions of the mitral valvular-ventricular complex in chronic ischemic mitral regurgitation. Circulation. 2003;108(suppl II):II116-21.

5. Langer F, Rodriguez F, Ortiz S, Cheng A, Nguyen TC, Zasio MK, et al. Subvalvular repair: the key to repairing ischemic mitral regurgitation? Circulation. 2005;112(suppl I):I383-9. 


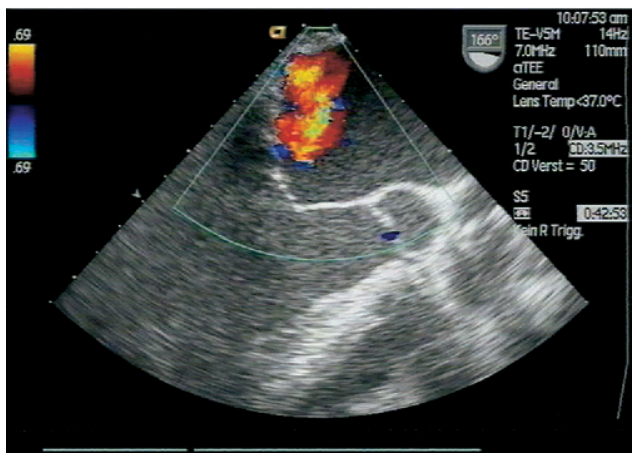

Figure E1. Intraoperative transesophageal echocardiography before cardiopulmonary bypass.

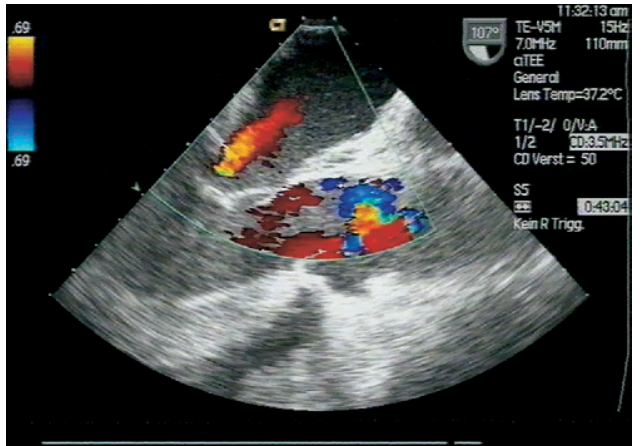

Figure E2. Intraoperative transesophageal echocardiography after undersized ring annuloplasty and before tightening of the transventricular suture.

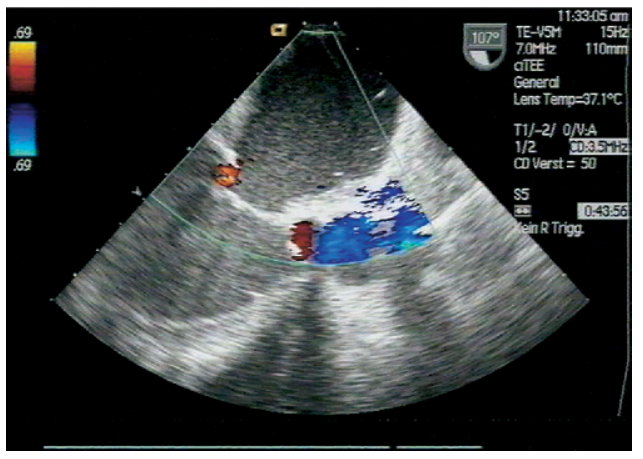

Figure E3. Intraoperative transesophageal echocardiography after undersized ring annuloplasty and after tightening of the transventricular suture. 
TABLE E1. Mitral regurgitation: Preoperative data, intraoperative data after RING and before STRING tightening, and intraoperative data after RING plus STRING

\begin{tabular}{lccc}
\hline $\begin{array}{l}\text { Patient } \\
\text { no. }\end{array}$ & Preoperative & $\begin{array}{c}\text { After RING, } \\
\text { before STRING }\end{array}$ & $\begin{array}{c}\text { RING plus } \\
\text { STRING }\end{array}$ \\
\hline 1 & 3 & 1 & 0 \\
2 & 3.5 & 2 & 1.5 \\
3 & 3 & 1 & 0.5 \\
4 & 3 & 1.5 & 1 \\
5 & 3 & 1 & 0 \\
6 & 4 & 2 & 1 \\
7 & 3 & 0 & 0 \\
8 & 3.5 & 1.5 & 1 \\
9 & 4 & 2 & 1 \\
10 & 3.5 & 0 & 1 \\
11 & 3 & 0.5 & 0 \\
12 & 3 & $1.2 \pm 0.8$ & $0.6 \pm 0.6^{*}$ \\
Mean & $3.3 \pm 0.4^{*}$ & 2 & 0.0 \\
\hline
\end{tabular}

Repeated-measures analysis of variance: $P<.001$. Dunnett post-hoc test: $* P<.05$ versus after RING and before STRING. RING, undersized ring annuloplasty; STRING, transventricular suture technique. 